\title{
Progresso temporal da brusone do trigo em função do inóculo primário, da aplicação de fungicida e da resistência dos genótipos
}

\author{
Delineide Pereira Gomes ${ }^{1}$, Valterley Soares Rocha ${ }^{2}$, João Romero Amaral Santos de Carvalho Rocha², Moacil Alves \\ de Souza ${ }^{2}$, Olinto Liparini Pereira ${ }^{2}$
}

${ }^{1}$ Instituto Federal do Maranhão, IFMA, Campus São Luis-Maracanã, 65095-460 - São Luis, MA, Brasil; ${ }^{2}$ Universidade Federal de Viçosa, Campus de Viçosa, Av. P.H Rolfs, s/n, 36570-900 - Viçosa, MG, Brasil.

Autor para correspondência: Delineide Pereira Gomes (delineide.gomes@ifma.edu.br).

Data de chegada: 02/11/2017. Aceito para publicação em: 15/07/2018.

$10.1590 / 0100-5405 / 187354$

\section{RESUMO}

Gomes, D.P.; Rocha, V.S.; Rocha, J.R.A.S.C.; Souza, M.A.; Pereira, O.L. Progresso temporal da brusone do trigo em função do inóculo primário, da aplicação de fungicida e da resistência dos genótipos. Summa Phytopathologica, v.45, n.1, p.50-58, 2019.

No Brasil, existem poucos estudos sobre a epidemiologia da brusone do trigo, consequentemente, pouca informação tem sido gerada sobre o progresso da doença. Este estudo avaliou o progresso temporal da brusone do trigo em quatro genótipos, submetidos ao controle químico e a diferentes quantidades de inóculo primário de Pyricularia graminis-tritici no campo. Os genótipos BRS 264, VI 98053, CD 116 e CD 104 foram inoculados com cinco volumes da suspensão fúngica (concentração de $1,5 \times 10^{5}$ esporos $\mathrm{mL}^{-1}$ ), de modo a obter, respectivamente, cinco porcentagens $(0,5,10,20$ e $30 \%)$ de plantas inoculadas na unidade experimental. A intensidade da brusone foi quantificada pela incidência e pela severidade da brusone em espigas e em folhas bandeira. As avaliações foram realizadas aos 1, 5, 10, 15, 20, 25, 30, 35, 40, 45, 50 dias após a inoculação (DAI). As equações de regressão foram significativas e ajustadas ao modelo logístico. A aplicação de fungicida (piraclostrobina + epoxiconazol) retardou a manifestação dos sintomas da brusone nos genótipos e, quando combinado com a cultivar moderadamente resistente (CD 116), teve melhor eficiência na redução da doença. As cultivares BRS 264 e CD 104 apresentam maior incidência e severidade em espigas no tempo. Em geral, os genótipos apresentam baixa severidade da brusone em folhas bandeira $(<0,45 \%)$ e com um baixo progresso temporal, nas diferentes quantidades de inóculo de $P$. graministritici. Na região de Viçosa-MG, nas condições de inóculo artificial estudadas, a cultivar $\mathrm{CD} 116$ se confirma como moderadamente resistente à brusone e os genótipos BRS 264, VI 98053 e CD 104 como suscetíveis.

Palavras-chave: Triticum aestivum, Pyricularia graminis-tritici, incidência, severidade, epidemiologia

\section{ABSTRACT}

Gomes, D.P.; Rocha, V.S.; Rocha, J.R.A.S.C.; Souza, M.A.; Pereira, O.L. Temporal progression of wheat blast as a function of primary inoculum, fungicide application and genotype resistance. Summa Phytopathologica, v.45, n.1, p.50-58, 2019.

In Brazil, there are few studies on the epidemiology of wheat blast; consequently, little information has been generated about the disease progress. This study evaluated the temporal progression of wheat blast in four genotypes subjected to chemical control and to different amounts of primary inoculum of Pyricularia graminis-tritici in the field. The genotypes BRS 264, VI 98053, CD 116 and CD 104 were inoculated with five volumes of fungal suspension (concentration of $1.5 \times 10^{5}$ spores. $\mathrm{mL}^{-1}$ ), in order to obtain, respectively, five percentages $(0,5,10,20$ and $30 \%)$ of plants inoculated in the experimental unit. The intensity of blast was quantified based on the incidence and the severity of this disease in spikes and flag leaves. Evaluations were performed at 1, 5, 10, 15, $20,25,30,35,40,45,50$ days after inoculation (DAI). The obtained regression equations were significant and adjusted to the logistic model. Application of the fungicide (pyraclostrobin plus epoxiconazole) delayed the onset of blast symptoms in the genotypes and, when combined with the moderately resistant cultivar (CD 116), had better efficiency in reducing the disease. The cultivars BRS 264 and CD 104 presented higher incidence and severity in the spikes according to time. In general, the genotypes present low severity of blast in flag leaves $(<0.45 \%)$ and a low temporal progression in the different amounts of inoculum of $P$. graminis-tritici. In the region of Viçosa-MG, Brazil, under the studied conditions of artificial inoculum, the cultivar CD 116 is confirmed as moderately resistant to blast and the genotypes BRS 264, VI 98053 and CD 104 are susceptible.

Keywords: Triticum aestivum, Pyricularia graminis-tritici, incidence, severity, epidemiology

A região central do Brasil tem grande potencial para a expansão tritícola, por apresentar condições diferenciadas de clima e solo, comparado às regiões tradicionais de cultivo (1), e nas últimas décadas, apresentou resultados muito promissores, principalmente pela possibilidade da utilização dos sistemas de irrigação $(1,5)$. No entanto, a produção de trigo ness a região tem sofrido danos significativos ocasionados pela brusone (13). Genótipos cultivados em Patos de Minas- MG, apresentaram incidências de até $89 \%$ e severidade de até $76 \%$ em espigas, cujo dano foi a redução na produtividade, a qual variou de 2,5 a 3,5 $\mathrm{kg} \mathrm{ha}^{-1}$, representando perdas de até $80 \%$ (5).

No Brasil, são limitados os estudos sobre a quantificação do progresso da brusone do trigo em campo, consequentemente, são poucas as informações geradas sobre a epidemiologia e medidas de controle genético e químico. A variabilidade genética para a resistência à brusone ainda é pouco explorada e conhecida, e os fungicidas atualmente recomendados não possuem a eficiência desejada devido à diversidade de isolados, com diferenças nos padrões de virulência, nos níveis de susceptibilidade das cultivares $(23,28)$ e nas condições ambientais dos 
locais de cultivo. Por isso, a brusone do trigo ainda é de difícil controle $(2,5,23)$. Entretanto, maior eficácia de controle para a brusone já foi obtida quando cultivares com grau moderado de resistência e o controle químico são combinados, com redução na incidência e na severidade da doença, e na taxa de progresso dos sintomas em espigas $(19,21,22,27)$.

A brusone do trigo, cujo agente causal nessa espécie obteve recentemente nova nomenclatura - Pyricularia graminis-tritici, determinada por meio de análises filogenéticas de diferentes isolados de trigo, arroz e outras gramíneas (4), apresenta, em condições naturais de cultivo, normalmente, sintomas mais em espigas, as quais apresentam coloração esbranquiçada ou descoloridas de sua metade superior (5, 13), todavia, podem ocorrer sintomas em outras partes da planta, como nas folhas (7) e na ráquis. A importância epidemiológica do estudo dos sintomas nas folhas para o desenvolvimento da brusone nas espigas ainda não foi elucidada (22), sendo hoje um componente bastante avaliado nos estudos de resistência à doença $(21,22,25)$.

Nesse contexto, as curvas de progresso temporal se tornam a melhor representação de uma epidemia (16), e as informações epidemiológicas obtidas ampliam o entendimento de como a doença se manifesta no hospedeiro e como outros fatores influenciam no seu desenvolvimento (24). Conforme Alves \& Fernandes (2) estudos epidemiológicos sobre esse patossistema são importantes para o estabelecimento de medidas de controle, principalmente quando se busca modelos de previsão que visam à tomada de decisão para a aplicação de fungicidas. Conforme esses autores, esses dados podem ser obtidos mais facilmente em experimentos sob condições controladas, mas podem não refletir o comportamento que ocorre sob condições naturais.

Nesse contexto, o objetivo deste estudo foi avaliar o progresso temporal da brusone do trigo em genótipos com diferentes reações à brusone, submetidos ao controle químico e a diferentes quantidades de inóculo primário de $P$. graminis-tritici (porcentagens de plantas inoculadas) no campo.

\section{MATERIAL E MÉTODOS}

O isolado de Pyricularia graminis-tritici foi obtido de grãos de trigo da cultivar MGS Brilhante, com $11 \%$ de incidência, produzida em Campos Altos, MG, Brasil. Após o isolamento, o fungo foi cultivado em meio de cultura batata, dextrose e ágar (BDA) e mantido sob fotoperíodo de $12 \mathrm{~h}$ de luz fluorescente a $26^{\circ} \mathrm{C}$, em câmara de incubação (BOD). Após a obtenção da colônia pura, foi realizada uma repicagem em meio de aveia (60 g de farinha de aveia, $12 \mathrm{~g}$ de ágar em $1 \mathrm{~L}$ de água) para proporcionar melhor esporulação. As placas com o fungo foram incubadas a $26^{\circ} \mathrm{C}$ e regime de $12 \mathrm{~h}$ de luz em câmara BOD durante dez dias. Após esse período, o micélio superficial foi retirado e colocado sob luz fluorescente constante à temperatura de $26{ }^{\circ} \mathrm{C}$ durante quatro dias para esporular (28). $\mathrm{O}$ isolado foi testado quanto à patogenicidade. Para isto, foi preparada uma suspensão de conídios $\left(1,0 \times 10^{5}\right.$ esporos $\mathrm{mL}^{-1}$ ) e inoculada em folhas de plântulas de trigo cultivar BRS 254, a qual apresentou sintomas e sinais do fungo.

Os conídios foram coletados das placas por adição de água destilada estérilizada, e a suspensão foi filtrada através de camadas de gaze e papel filtro esterilizados. A suspensão de esporos foi calibrada após determinação da densidade em hemacitômetro, obtendo-se concentração de $1,5 \times 10^{5}$ esporos $\mathrm{mL}^{-1}$, acrescida de Tween $20(0,01 \%)$.

$\mathrm{O}$ experimento em campo foi realizado em Viçosa, MG, Brasil, localização de $20^{\circ} 45^{\prime} \mathrm{LS}, 42^{\circ} 51^{\prime} \mathrm{W}$ e, altitude de $651 \mathrm{~m}$, em Argissolo Vermelho Amarelo. O tipo de clima é CWA, e a umidade relativa do ar média de $80 \%$. Os cultivos anteriores da área experimental não tinham histórico de brusone, e nem nas plantas daninhas lá existentes, em especial, gramíneas nativas ou invasoras.

Foram utilizados quatro genótipos de trigo com as seguintes reações à brusone: BRS 264, como suscetível (19); CD 116, como moderadamente resistente (11); VI 98053 e CD 104, ambas sem informação quanto à resistência em Minas Gerais, Brasil.

A semeadura e o manejo dos genótipos foram realizados conforme as recomendações técnicas para a região (11). A semeadura foi realizada na primeira quinzena de maio de 2012 , a $19,5^{\circ} \mathrm{C}$ e $83 \%$ de umidade relativa do ar. O cálculo da taxa de semeadura foi condicionado ao peso da semente, germinação e quantidade de sementes por metro de sulco. O cultivo foi irrigado por aspersão convencional.

O delineamento experimental foi o de blocos casualizados, com três repetições por tratamento, totalizando 60 parcelas. Cada parcela foi composta por cinco linhas de 5,0 m de comprimento, e as linhas separadas por 0,20 m. Entre as parcelas no mesmo bloco foi obedecida a distância de 1,5 m. Foram consideradas, para fins de área útil, as três linhas centrais da parcela, desconsiderando-se $30 \mathrm{~cm}$ em cada extremidade da linha.

Antes da inoculação, as plantas foram molhadas por meio de irrigação por aspersão por dez minutos. As inoculações iniciaram com as espigas das completamente emergidas, correspondendo aos estádios 58-60 da escala de Zadoks et al. (30). Devido às diferenças no ciclo dos genótipos, a inoculação foi escalonada.

As inoculações foram realizadas a partir das 17 h, em condições semelhantes de temperatura e umidade do ar, sem precipitação, conforme Goulart et al. (13). Foram registradas a temperatura e a umidade do ar após a inoculação (Figura 1).

Em cada genótipo, os tratamentos foram obtidos a partir da aplicação de cinco volumes $(0 ; 0,20 ; 0,35 ; 0,70$ e 1 L) da suspensão de

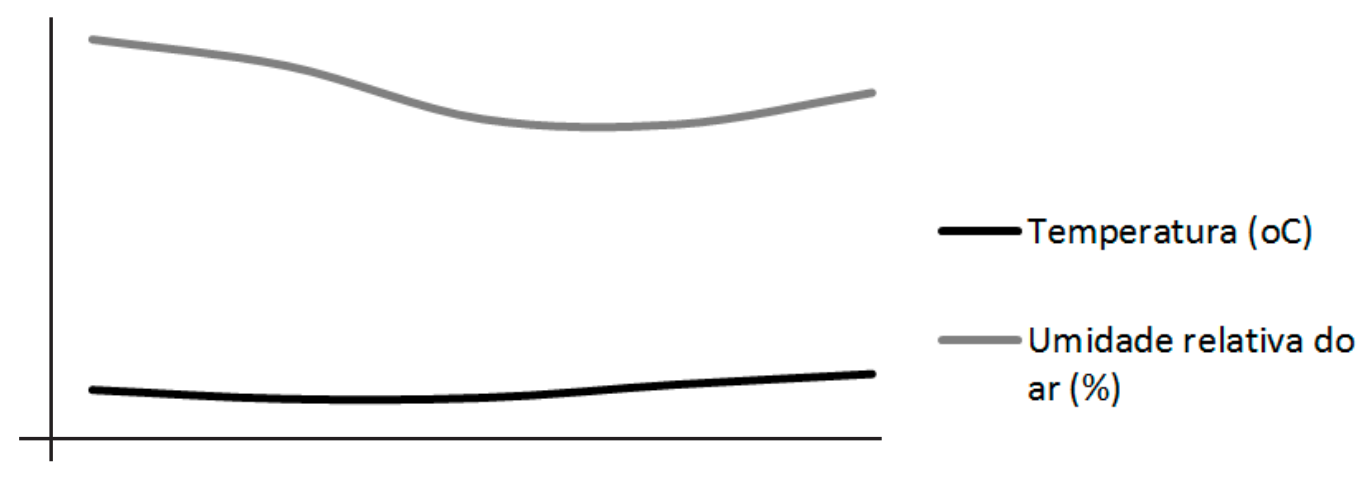

Figura 1. Condições ambientais após a inoculação dos genótipos BRS 264, VI 98053, CD 116 e CD 104, durante o ensaio em Viçosa-MG, Brasil 
conídios (concentração padrão de $1,5 \times 10^{5}$ esporos $\mathrm{mL}^{-1}$ ) de $P$. graministritici. Esses volumes foram calculados e testados para inocular de maneira satisfatória, respectivamente, cinco porcentagens de plantas da unidade experimental $(0,5,10,20$ e $30 \%)$, ou seja, os volumes de $0 ; 0,20 ; 0,35 ; 0,70$ e $1 \mathrm{~L}$ da suspensão de conídios foram aplicados de modo a obter os tratamentos de $0,5,10,20$ e $30 \%$ de plantas inoculadas na unidade experimental, respectivamente.

O volume zero (sem inóculo) correspondeu à aplicação de água, seguido do controle químico a base de piraclostrobina + epoxiconazol na dose de $0,5 \mathrm{~L} \mathrm{ha}^{-1}$ do produto comercial. Os tratamentos foram aplicados com pulverizador de $2 \mathrm{~L}$, pressão de 3,0 bar (43,5 psi), de forma homogênea e uniforme, até o escorrimento nas plantas.

No momento da inoculação, cada parcela/tratamento foi isolada fisicamente da parcela vizinha, por meio de lonas plásticas lavadas, com a finalidade de se manter um microclima favorável ao patógeno e também para se evitar a deriva do inóculo, sendo essa proteção retirada $72 \mathrm{~h}$ após a inoculação.

Após a inoculação, a presença de água livre sobre folhas e espigas foi mantida por $12 \mathrm{~h}$ (período de molhamento) por meio da irrigação por aspersão de baixa pressão, e isto foi auxiliado pelo microclima proporcionado pelo orvalho e pelas zonas de concentração de umidade no solo na área experimental.

Para a avaliação da intensidade da brusone foram analisadas: incidência de $P$. graminis-tritici nas espigas, severidade da brusone em espigas e em folhas bandeira. A incidência foi quantificada nas plantas da área útil (três linhas centrais da parcela), com base na quantificação dos sintomas na espiga. A severidade foi obtida pela análise de 30 plantas tomadas ao acaso na área útil, utilizando a escala diagramática apropriada para a quantificação dos sintomas na espiga (15) e na folha bandeira (18). As notas da severidade em espigas e em folhas bandeira foram dadas com base na intensidade destes sintomas nessas 30 plantas, e por semelhança com os valores da escala.

As avaliações da incidência e da severidade da doença foram realizadas em intervalos de cinco dias a partir da inoculação, com avaliações aos $1,5,10,15,20,25,30,35,40,45$ e 50 dias após a inoculação (DAI), sendo a avaliação final aos 50 DAI. A partir desses dados, foram elaboradas as curvas de progresso da doença.

Foram realizadas análises de regressão pelo PROC NLIN, e análises de correlação, pelo PROC CORR, ambos por meio do software estatístico SAS®. A escolha do modelo mais adequado foi baseada na análise de parâmetros de qualidade do ajuste, tais como o quadrado médio do resíduo, coeficiente de determinação e gráficos dos resíduos. As áreas abaixo da curva de progresso para a incidência, severidade da brusone na espiga e severidade da brusone na folha bandeira foram obtidas pelo PROC GLM, software SAS ${ }^{2}$. Os dados foram comparados pelo teste de Tukey, $\mathrm{p} \leq 0,05$.

\section{RESULTADOS E DISCUSSÃO}

Para todas as variáveis analisadas, as equações foram significativas e ajustadas ao modelo logístico, como aquele que melhor se ajustou, com coeficientes de regressão $\left(\mathrm{R}^{2}\right)$ variando de 91 a $99 \%(\mathrm{p}<0,001)$ (Figuras 2 a 6).

Até aos 20-25 dias após a inoculação (DAI), houve um atraso na incidência de $P$. graminis-tritici nos genótipos tratados com fungicida (Figura 2). Aproximadamente aos 25 DAI, a doença se iniciou nos genótipos BRS 264 e CD 104; após os 30 DAI essa se iniciou para a linhagem VI 98053; e aos 40 DAI se iniciou para a cultivar CD 116.

A aplicação do fungicida retardou a severidade da brusone nas espigas em todos os genótipos (Figura 3). No entanto, na cultivar CD 104, os sintomas evoluíram mais cedo, alcançando aproximadamente $20 \%$ de severidade na espiga já aos 20 DAI.

Para o genótipo CD 116 foi obtida a menor AACPI, para as diferentes porcentagens de inóculo (\% de plantas inoculadas) (Tabela 1). Isso pode ser explicado pela resistência moderada à brusone apresentada por essa cultivar (12).

A aplicação do fungicida mais a resistência da cultivar CD 116 reduziu a incidência e a severidade da doença em espigas, em comparação com a aplicação do fungicida isoladamente (cultivares suscetíveis tratadas) ou comparado com a resistência isoladamente (cultivar CD 116 sem controle). A eficiência da combinação dessas duas medidas também foi demonstrada por outros pesquisadores (19, $21,22,27)$.

Em geral, as cultivares moderadamente resistentes podem ser eficientes em condições de baixa ou média intensidade da brusone. No entanto, em condição de alta intensidade da doença, esta medida deve ser complementada com semeadura em épocas adequadas (20) e

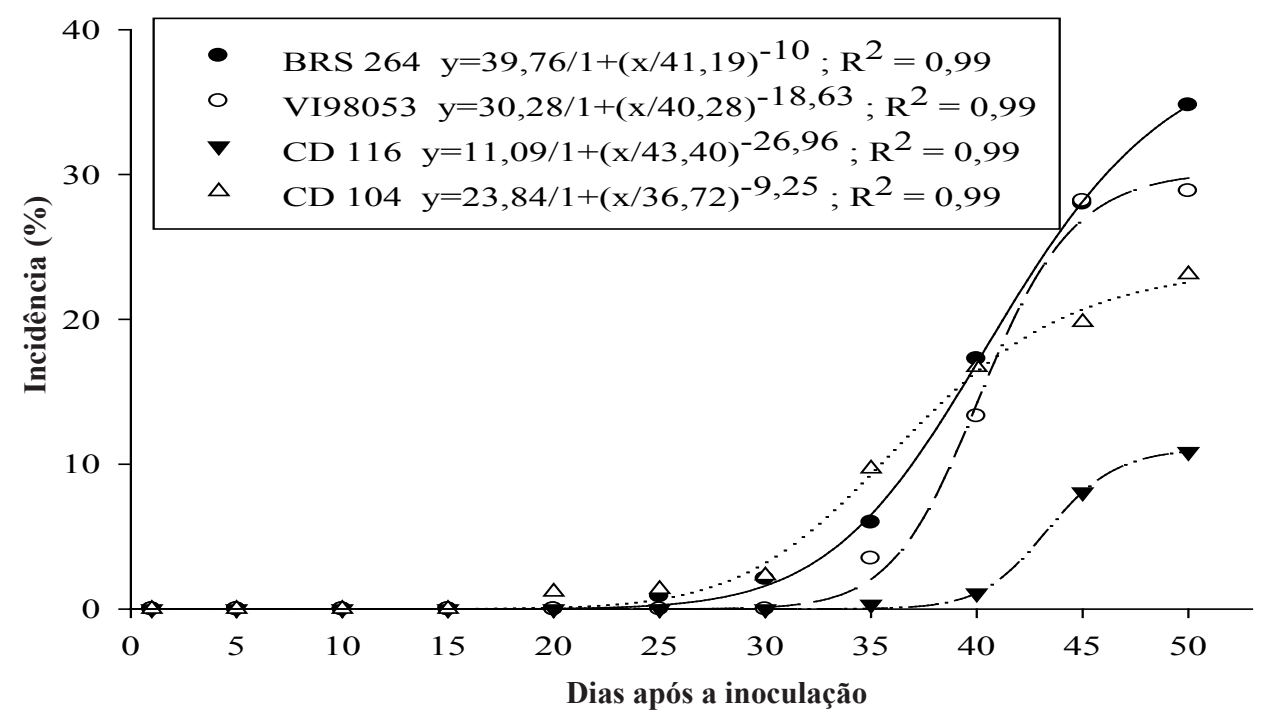

Figura 2. Progresso temporal da incidência da brusone em espigas de genótipos de trigo com diferentes reações à doença e tratados com fungicida 


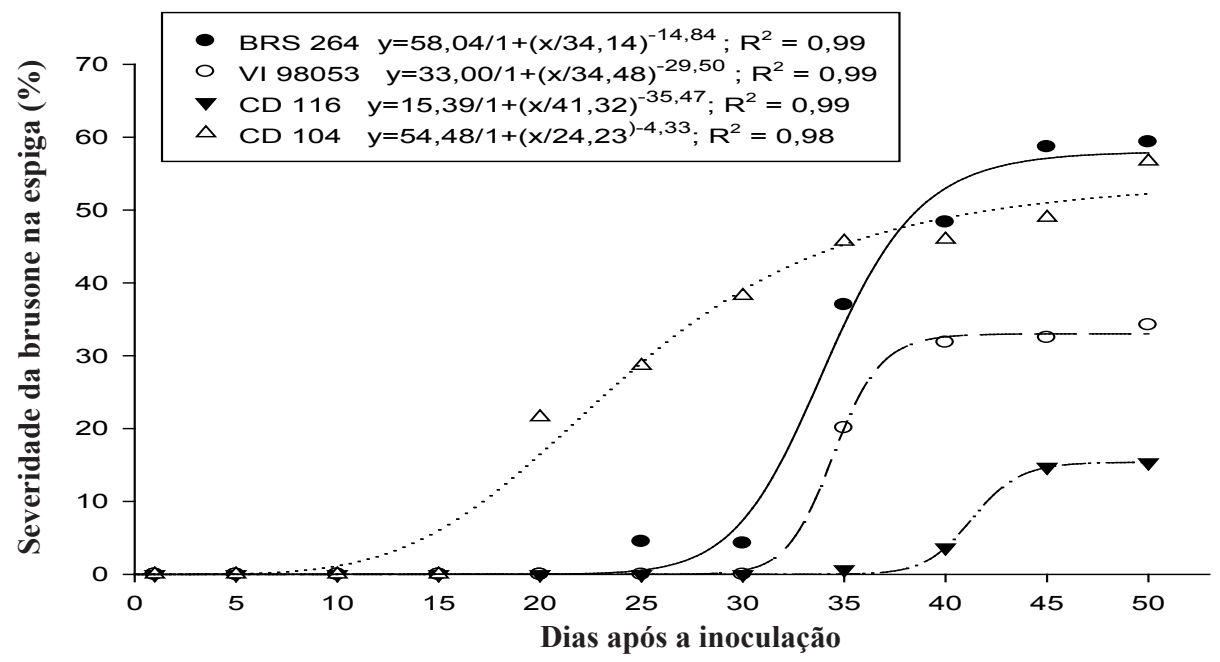

Figura 3. Progresso temporal da severidade da brusone em espigas de genótipos de trigo com diferentes reações à doença e tratados com fungicida
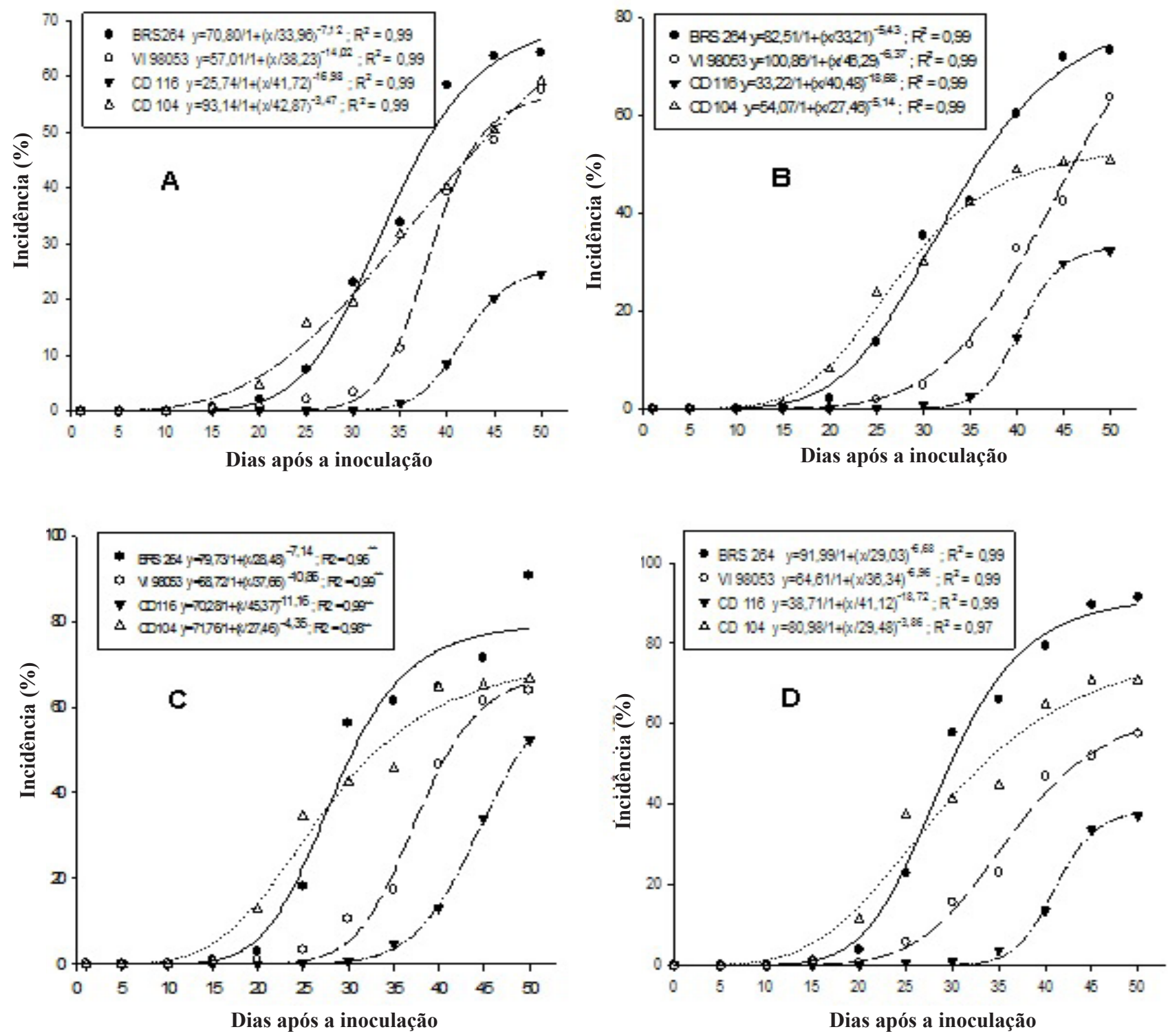

Figura 4. Progresso temporal da incidência da brusone em espigas de genótipos de trigo com diferentes reações à doença, submetidos a 5\% (A), $10 \%$ (B), 20\% (C) e 30\% (D) de plantas inoculadas com Pyricularia graminis-tritici no campo 

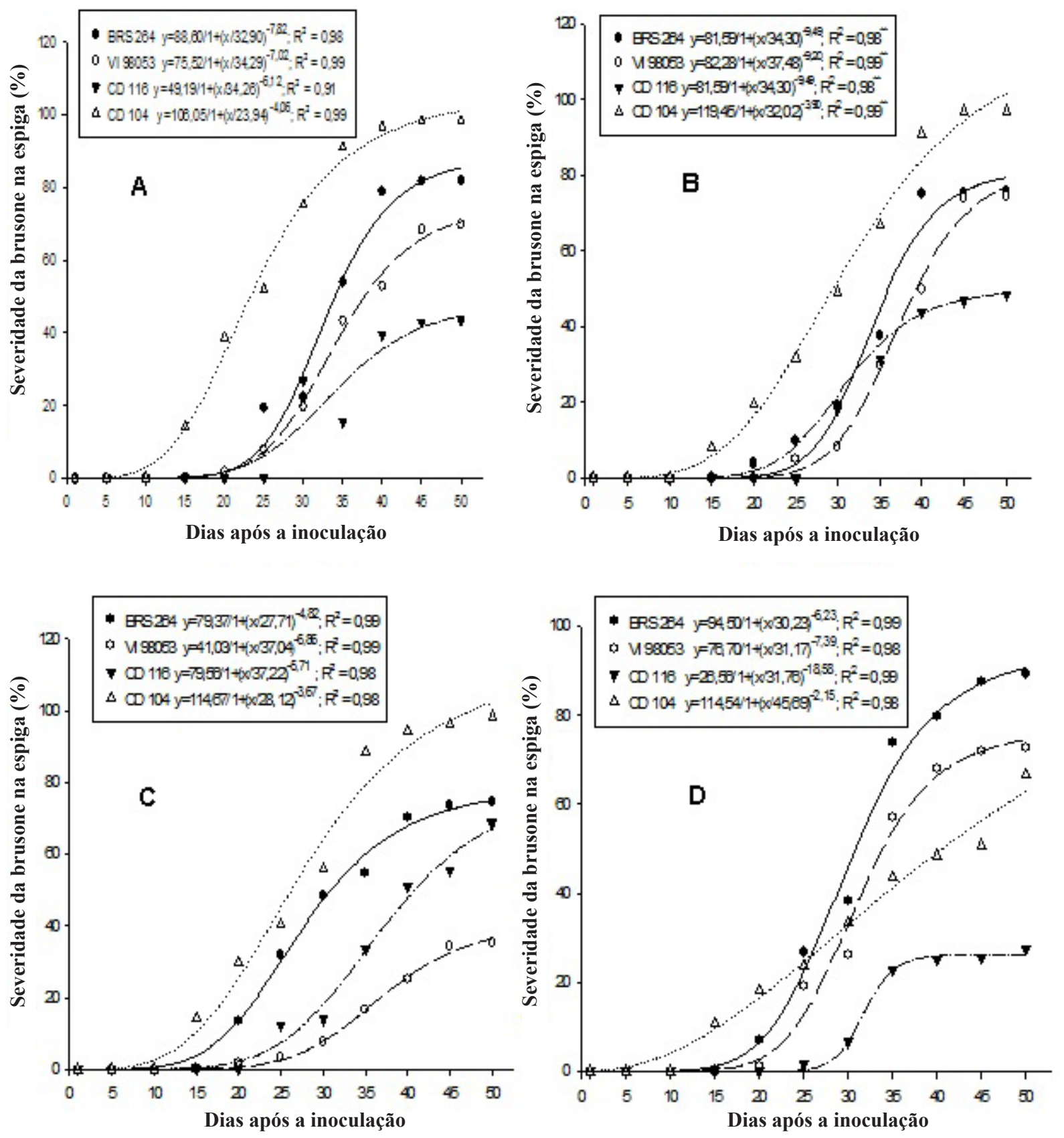

Figura 5. Progresso temporal da severidade da brusone em espigas de genótipos de trigo com diferentes reações à doença, submetidos a 5 \% (A), $10 \%$ (B), $20 \%$ (C) e $30 \%$ (D) de plantas inoculadas com Pyricularia graminis-tritici no campo

aplicação de fungicidas (19), como visto aqui.

As cultivares BRS 264 e CD 116 apresentaram, respectivamente, a maior e a menor incidência de $P$. graminis-tritici no tempo (Figura 4). No entanto, nas diferentes quantidades de inóculo, a doença se manifestou mais cedo para a cultivar CD 104. Em geral, a incidência da brusone progride consideravelmente no tempo, a partir de uma fonte de inóculo primário conhecida (22).

A cultivar CD 104, nos diferentes tratamentos inoculados, também apresentou severidade na espiga mais cedo em relação aos demais genótipos, já entre 8-10 DAI (Figura 5), e também, numericamente, os maiores valores de AACPSE (Tabela 1), mas com poucas diferenças. Para a maioria das quantidades de inóculo, essa cultivar apresentou maior progresso temporal da severidade da brusone em espigas (Figura $5)$.

A severidade da brusone em folhas bandeira não foi observada nos genótipos VI 98053, CD 116 e CD 104 quando utilizado o controle químico. No entanto, a cultivar BRS 264 apresentou severidade na folha de $0,03 \%$ aos 40 DAI, mesmo com o controle.

Em geral, os genótipos não diferiram quanto à área abaixo da curva de progresso para a severidade da brusone em folhas bandeira 

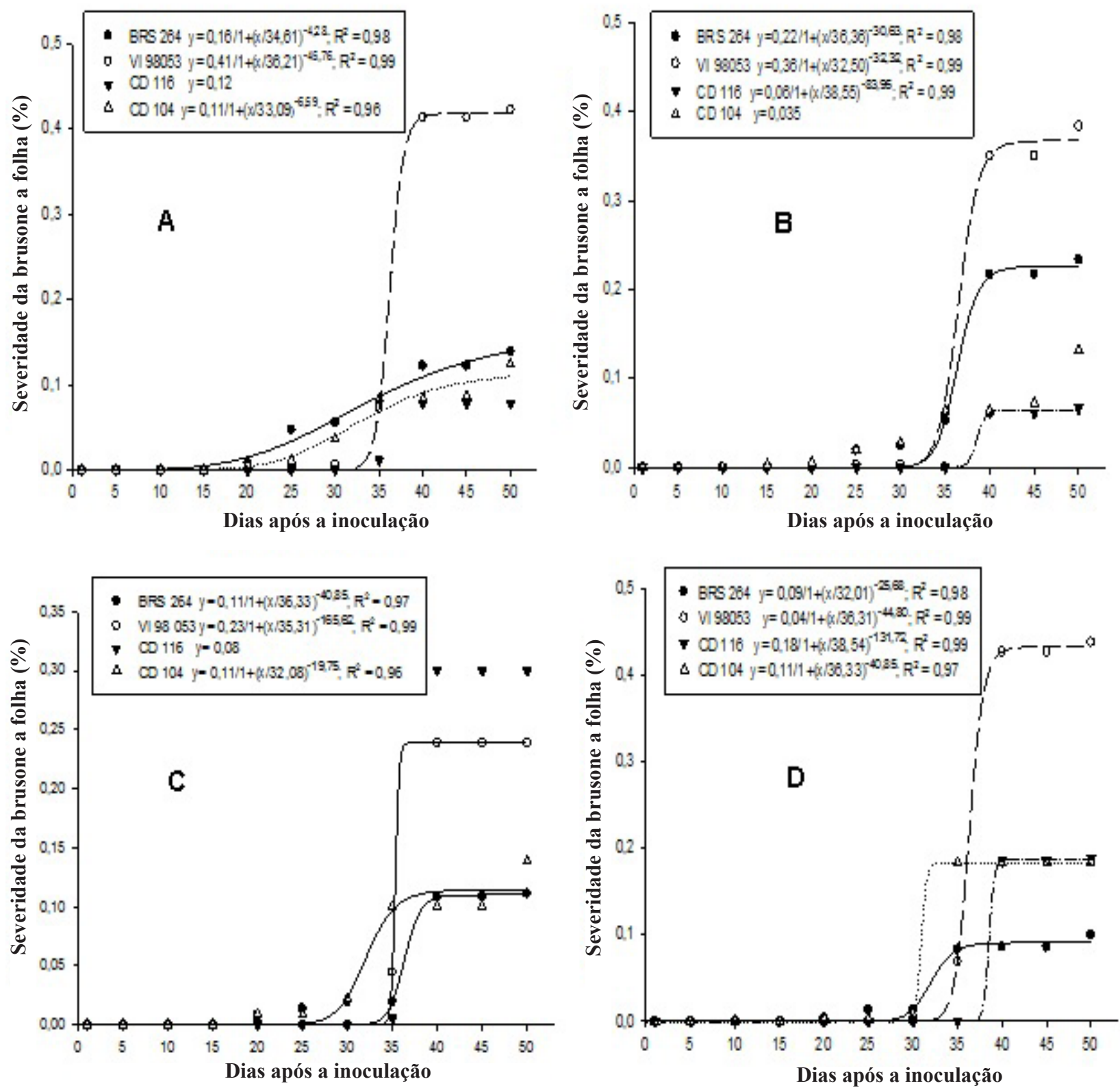

Figura 6. Progresso temporal da severidade da brusone em folhas bandeira de genótipos de trigo com diferentes reações à doença, submetidos a $5 \%$ (A), $10 \%$ (B), $20 \%$ (C) e $30 \%$ (D) de plantas inoculadas com Pyricularia graminis-tritici no campo

\section{(AACPSF) (Tabela 1).}

Para a cultivar BRS 264, houve correlações positivas entre a incidência e a severidade da brusone em espigas e em folhas bandeira (Tabela 2). Por isso, a presente pesquisa confirma a alta suscetibilidade da cultivar BRS 264 à brusone na região, o que corrobora com Pagani et al. (19). Por outro lado, Coelho et al. (5) afirmam que essa cultivar pode ser tolerante, mesmo quando exposta à alta intensidade da doença. Esses verificaram, em espigas, incidência de $66 \%$ e severidade de 53 $\%$ nessa cultivar.

Nos genótipos foram constatadas baixas porcentagens de severidade da brusone em folhas bandeira $(<0,45 \%)$ (Figura 6$)$. No entanto, após 35 DAI, a linhagem VI 98053 apresentou perceptíveis acréscimos, com valores entre $0,2-0,4 \%$ nas diferentes quantidades de inóculo. Ressaltase, que esse genótipo apresentou correlação positiva entre a incidência em espigas e a severidade da brusone em folhas bandeira (Tabela 2).

Além dos baixos valores de severidade da brusone em folhas bandeira, foi constatado também o baixo progresso temporal da doença nesse órgão, com pontos de estabilização, para as diferentes quantidades de inóculo (Figura 6). Para a cultivar BRS 264, o inicio se deu após os 30 DAI, e sua estabilização, aproximadamente, aos 40 DAI, nas quantidades de 10,20 e $30 \%$ de plantas inoculadas. Para a linhagem VI 98053, o inicio se deu entre 32-34 DAI e a estabilização entre 37-40 DAI, em todas as quantidades de inóculo. Estes curtos intervalos de tempo entre o inicio e a estabilização caracterizam o baixo progresso da severidade da doença nas folhas bandeira.

Urashima et al. (29) não observaram sintomas da brusone nas folhas da cultivar CD 104, diferindo da presente pesquisa com a mesma cultivar. Possivelmente, isso se relaciona com a baixa incidência da 
Tabela 1. Área abaixo da curva de progresso da incidência da brusone em espigas (AACPI), da severidade da brusone em espigas (AACPSE) e da severidade da doença em folhas bandeira (AACPSF) de genótipos de trigo inoculados com diferentes quantidades de inóculo de Pyricularia graminis-tritici no campo

\begin{tabular}{|c|c|c|c|c|}
\hline Genótipo de trigo & $\begin{array}{l}\text { Quantidade de inóculo } \\
\text { (\% de plantas inoculadas) }\end{array}$ & $\begin{array}{c}\text { Incidência nas espigas } \\
\text { (AACPI) }\end{array}$ & $\begin{array}{c}\text { Severidade em espigas } \\
\text { (AACPSE) }\end{array}$ & $\begin{array}{l}\text { Severidade em folhas } \\
\text { bandeira (AACPSF) }\end{array}$ \\
\hline BRS 264 & & $6.5 \mathrm{a}$ & $9.54 \mathrm{a}$ & $0.007 \mathrm{a}$ \\
\hline CD 116 & (+ fungicida) & $1.4 \mathrm{~b}$ & $2.5 \mathrm{a}$ & $0 \mathrm{a}$ \\
\hline CD 104 & & $5.4 \mathrm{a}$ & $19.8 \mathrm{a}$ & $0 \mathrm{a}$ \\
\hline BRS 264 & & $18.7 \mathrm{a}$ & $25.1 \mathrm{ab}$ & $0.03 \mathrm{a}$ \\
\hline CD 116 & & $3.9 \mathrm{c}$ & $12.1 \mathrm{~b}$ & $0.01 \mathrm{a}$ \\
\hline CD 104 & & $16.2 \mathrm{ab}$ & $39.2 \mathrm{a}$ & $0.03 \mathrm{a}$ \\
\hline BRS 264 & & $21.9 \mathrm{a}$ & $15.3 \mathrm{a}$ & $0.05 \mathrm{a}$ \\
\hline VI 98053 & 10 & $11.3 \mathrm{~b}$ & $18.0 \mathrm{a}$ & $0.08 \mathrm{a}$ \\
\hline CD 116 & & $7.3 \mathrm{c}$ & $16.4 \mathrm{ab}$ & $0.06 \mathrm{a}$ \\
\hline CD 104 & & $23.6 \mathrm{ab}$ & $36.3 \mathrm{a}$ & $0.03 \mathrm{a}$ \\
\hline BRS 264 & & $30.0 \mathrm{a}$ & $23.5 \mathrm{a}$ & $0.02 \mathrm{a}$ \\
\hline VI 98053 & 30 & $14.8 \mathrm{c}$ & $29.0 \mathrm{a}$ & $0.1 \mathrm{a}$ \\
\hline CD 116 & & $6.5 \mathrm{~d}$ & $8.1 \mathrm{a}$ & $0.04 \mathrm{a}$ \\
\hline CD 104 & & $22.6 \mathrm{~b}$ & $22.8 \mathrm{a}$ & $0.05 \mathrm{a}$ \\
\hline
\end{tabular}

Médias seguidas de mesma letra na coluna, para cada porcentagem de plantas inoculadas, não diferem pelo teste de Tukey ao nível de 5\% de probabilidade.

Tabela 2. Coeficientes de correlação de Pearson obtidos entre as variáveis de intensidade da brusone avaliados em genótipos de trigo inoculados com Pyricularia graminis-tritici em campo

\begin{tabular}{|c|c|c|c|}
\hline Genótipo & & Severidade em espigas & Severidade em folhas bandeira \\
\hline \multirow[b]{2}{*}{ BRS 264} & Incidência de $P$. graminis-tritici & $0,88^{*}$ & $0,95^{*}$ \\
\hline & Severidade da brusone na espiga & - & $0,93 *$ \\
\hline \multirow[b]{2}{*}{ VI 98053} & Incidência de $P$. graminis-tritici & $0,03^{\mathrm{ns}}$ & $0,94 *$ \\
\hline & Severidade da brusone na espiga & - & $0,29^{\text {ns }}$ \\
\hline CD 116 & Severidade da brusone na espiga & - & $0,40^{\text {ns }}$ \\
\hline \multirow[b]{2}{*}{ CD104 } & Incidência de $P$. graminis-tritici & $0,69^{\text {ns }}$ & $0,39^{\text {ns }}$ \\
\hline & Severidade da brusone na espiga & - & $0,15^{\mathrm{ns}}$ \\
\hline
\end{tabular}

*Significativo ao nível de $5 \%$ de probabilidade; ${ }^{\text {ns }}$ - Não significativo ao nível de $5 \%$ de probabilidade pelo Teste t.

doença que ocorre em geral nas folhas de trigo.

Os resultados deste estudo são semelhantes ao relatado por Rocha et al. (23), os quais verificaram que a severidade da brusone em espigas foi maior do que a severidade em folhas bandeira; e que o controle com fungicidas foi eficiente nas folhas, mas não nas espigas. Provavelmente devido à baixa severidade que geralmente ocorre nas folhas.

Sugere-se que a penetração e a colonização nas folhas pelo fungo, bem como na ráquis, seja um atributo importante a ser investigado nos estudos de resistência do hospedeiro, pois envolvem componentes morfológicos ou estruturais da planta que podem ser utilizados como barreiras à entrada do patógeno $(3,25,26)$, tais como epiderme, parede celular ou tecidos de parênquima, além de componentes bioquímicos (8) ou físiológicos $(10,21)$.

Em relação aos componentes fisiológicos, o processo gravemente afetado é a fotossíntese (9) e a translocação de fotossintatos para as espigas. O patógeno, ao colonizar a folha, produz lesões que coalescem e necrosam o tecido (14), possibilitando perda de área foliar, o que interfere negativamente na fotossíntese (21). Na ráquis, além das lesões 
de proporções indefinidas, o fungo pode causar pontos de infecção que bloqueiam a translocação de fotossintatos para a espiga, impedindo o enchimento dos grãos, tornando-os "chochos" e com baixo peso específico (13). Além dos danos na formação da semente, o patógeno pode ser transmitido através dela (17).

Apesar do controle químico ter sido capaz de retardar a incidência e a severidade da doença nos tratamentos controle (Figuras 2 e 3), a eficiência não foi a almejada, uma vez que houve a produção de inóculo secundário a partir do inóculo primário oriundos das parcelas inoculadas, e com a dispersão de conídios através do vento promoveu-se a doença, mesmo nas parcelas tratadas. Isto corrobora com a literatura, pois o controle pela aplicação de piraclostrobina+ epoxiconazol ou pela mistura de outras estrobilurinas com triazóis ainda não atingiram a eficiência desejada (23).

No contexto do manejo integrado da doença, a aplicação preventiva de fungicidas já na fase vegetativa da cultura torna-se interessante, pois pode se reduzir a produção de inóculo nas folhas (6) e consequentemente reduzir a disseminação do fungo para a fase mais suscetível da cultura que é a reprodutiva, desde a formação da ráquis floral, o espigamento, até o enchimento dos grãos. Por exemplo, Urashima \& Kato (27) observaram que a aplicação dos fungicidas probenazol e triciclazol no controle da brusone do trigo proporcionou boa proteção no estádio vegetativo, mas não nas espigas.

No campo, a epidemiologia da brusone do trigo ainda é pouco estudada e compreendida, tornando mais difícil a recomendação da forma de controle ou manejo mais eficiente (22). Quanto à quantificação dessa doença, as pesquisas podem esclarecer em quais níveis de incidência e de severidade os fungicidas devem ser aplicados; bem como relatar em quais níveis da doença podem ocorrer a «quebra» da resistência; e por fim, o momento no qual se deve combinar medidas para o manejo integrado da doença.

Pelos resultados do presente estudo, conclui-se que o controle químico com piraclostrobina mais epoxiconazol é capaz de "atrasar" ou retardar a brusone nos genótipos BRS 264, VI 98053 e CD 104, e, quando combinado com uma cultivar moderadamente resistente (CD 116), tem melhor eficiência na redução nos índices da doença. As cultivares BRS 264 e CD 104 apresentam as maiores incidências da brusone em espigas (valores entre $65-97 \%$ para a cultivar BRS 264, e entre 51-69 \% para a cultivar CD 104), bem como, os maiores valores de severidade da doença em espigas no tempo (entre 79-92\% para a cultivar BRS 264, e entre 67-100 \% para a cultivar CD 104), nas condições de 5, 10, 20 e $30 \%$ de plantas inoculadas no campo. Os genótipos avaliados apresentam baixas porcentagens de severidade da brusone em folhas bandeira $(<0,45 \%)$, e com um baixo progresso temporal, com pontos de estabilização entre 35-40 DAI. Na região de Viçosa-MG, sob as condições de inóculo artificial avaliadas, as cultivares BRS 264 e CD 104 e a linhagem VI 98053 mostramse suscetíveis à brusone. E a cultivar CD 116 confirma-se como moderadamente resistente a doença.

\section{AGRADECIMENTOS}

A FAPEMIG (Fundação de Amparo e Pesquisa de Minas Gerais, Brasil) pelo apoio financeiro. Ao Sr. José Cupertino, técnico de laboratório da UFV, pelo auxílio na preparação da suspensão de conídios.

\section{REFERÊNCIAS}

1. Albrecht, J.C.; Vieira, E.; Silva, S.M.; Andrade, J.M.V.; Scheeren, P.L.; Trindade, M.G.; Sobrinho, J.S.; Sousa, C.N.A.; Reis, W.P.; Ribeiro Júnior, W.Q.; Fronza, V.; Cargnin, A.; Yamanaka, C.H. Adaptabilidade e estabilidade de genótipos de trigo irrigado no Cerrado do Brasil Central. Pesquisa Agropecuária Brasileira, Brasília, DF, v.42, n.12, p. 1727-1734, 2007.

2. Alves, K.J.P.; Fernandes, J.M.C. Influência da temperatura e da umidade relativa do ar na esporulação de Magnaporthe grisea em trigo. Fitopatologia Brasileira, Brasília, DF, v.31, n.6, p.579584, 2006.

3. Araújo, L.; Soares, J.M.; Filippi, M.C.C.D.; Rodrigues, F.A. Cytological aspects of incompatible and compatible interactions between rice, wheat and the blast pathogen Pyricularia oryzae. Scientia Agrícola, Piracicaba, v.73, n.2, p.177-183, 2016.

4. Castroagudin, V. L.; Moreira, S. I.; Pereira, D. A. S.; Moreira, S. S. ; Brunner, P.; Maciel, J. L. N.; Crous, P.; Mcdonald, B.A.; Alves, E.; Ceresini, P.C. Pyricularia graminis-tritici, a new Pyricularia species causing wheat blast. Persoonia, Netherlands, p. 199-216, 2016. Disponível em: https:// www.ncbi.nlm.nih.gov/pmc/articles/PMC5315288/Acesso em: 7 jan. 2017.

5. Coelho, M.A. de O.; Torres, G.A.M.; Cecon, P.R.; Santana, F.M. Sowing date reduces the incidence of wheat blast disease. Pesquisa Agropecuária Brasileira, Brasília, DF, v.51, n.5, p.631-637, 2016.

6. Cruz, C.D.; Kiyuna, J.; Bockus, W.W.; Todd, T.C.; Stack, J.P.; Valent, B. Magnaporthe oryzae conidia on basal wheat leaves as a potential source of wheat blast inoculum. Plant Pathology, London, v.64, n.6,p.1491-1498, 2015. Disponível em: <http://onlinelibrary.wiley.com/doi/10.1111/ ppa.12414/epdf>. Acesso em: 8 mar. 2016.

7. Cruz, M.F.A.; Rios, J.A.; Araujo, L.; Rodrigues, F.A. Infection process of Pyricularia oryzae on the leaves of wheat seedlings. Tropical Plant Pathology, Brasília, DF, v.41, n.2, p.123-127, 2016.

8. Debona, D.; Rodrigues, F.A.; Rios, J.A.; Telles, K.J.N. Biochemical changes in the leaves of wheat plants infected by Pyricularia oryzae. Phytopathology, St. Paul, v.102, n.12, p.1121-1129, 2012. Disponível em: <http:// apsjournals.apsnet.org/doi/pdf/10.1094/PHYTO-06-12-0125-R>. Acesso em: 10 jun. 2015.

9. Debona, D.; Rodrigues F.A,; Rios, J.A; Martins, S.C.V.; Pereira L,.F.; Matta, F.M. da. Limitations to photosynthesis in leaves of wheat plants infected by Pyricularia oryzae. Phytopathology, St. Paul, v.104, n.1, p.34-39, 2014. Disponível em: <http://apsjournals.apsnet.org/doi/pdf/10.1094/PHYTO01-13-0024-R>. Acesso em: 10 jun. 2015.

10. Debona, D.; Rios, J.A.; Nascimento, K.J.T.; Silva, L.C.; Rodrigues, F.A. Influence of magnesium on physiological responses of wheat infected by Pyricularia oryzae. Plant Pathology, London v.65, n.1, p.114-123, 2016. Disponível em: <http://onlinelibrary.wiley.com/doi/10.1111/ppa.12390/ pdf $>$. Acesso em: 7 jan. 2017.

11. Embrapa. Informações técnicas para trigo e triticale: safra 2012. In: Reunião da Comissão Brasileira de Pesquisa de Trigo e Triticale, 5., 2011, Dourados. Sistemas de produção, 9. Dourados, MS: Embrapa Agropecuária Oeste, 2011, 204 p. Disponível em:<http://www.cnpt.embrapa.br/culturas/trigo/ informacoes_tecnicas_trigo_triticale_safra_2012.pdf $>$. Acesso em: 01 mar. 2012.

12. Franco, A.F.; Marchioro, V.S.; Dalla Nora, T.; Schuster, I.; Oliveira, E.F.; Vieira, E.S.N.; Lima, F.J.A. CD 116: A healthy wheat cultivar with industrial quality. Crop Breeding and Applied Biotechnology, Londrina, v.9, n.2, p.196-198, 2009.

13. Goulart, A.C.P.; Sousa, P.G.; Urashima, A.S. Danos em trigo causados pela infecção de Pyricularia grisea. Summa Phytopathologica, Piracicaba, v.33, n.4, p.358-363, 2007

14. Islam, M. T.; Croll, D.; Gladieux, P.; Soanes, D. M.; Persoons, A.; Bhattacharjee, P.; Hossain, MS; Gupta, DR; Rahman, MM; Mahboob, MG; Cook, N; Salam, MU; Surovy, MZ; Sancho, VB; Maciel, JL; Nhani Júnior, A; Castroagudín, VL; Reges, JT; Ceresini, PC; Ravel, S; Kellner, R; Fournier, E; Tharreau, D; Lebrun, MH; McDonald, BA; Stitt, T; Swan, D; Talbot, NJ; Saunders, DG; Win, J; Kamoun, S. Emergence of wheat blast in Bangladesh was caused by a South American lineage of Magnaporthe oryzae. BMC biology, London, v.14, n.1, p.84, 2016.

15. Maciel, J.L.N.; Danelli, A.L.D.; Boaretto, C.; Forcelini, C.A. Diagrammatic scale for the assessment of blast on wheat spikes. Summa Phytopathologica, Botucatu, v.39, n.3, p.162-166, 2013.

16. Marcuzzo, L.L.; Santos, J.E. Progresso temporal do chumbinho em di- 
ferentes genótipos de pessegueiro. Summa Phytopathologica, Botucatu, v.43, n.3, p.239-242, 2017.

17. Menten, J.O.M.; Moraes, M.H.D. Importância da semente na disseminação de Pyricularia sp. na cultura do trigo. Summa Phytopathologica, Jaguariúna, v.14, n.1-2, p.53, 1988 (Resumo).

18. Notteghem, J.L. Cooperative experiment on horizontal resistance to rice blast. In: Blast and upland rice: report and recommendations from the meeting for international collaboration in upland rice improvement. International Rice Research Institute: Los Baños, Filipinas, 1981. p.43-51.

19. Pagani, A.P.S.; Dianese, A.C.; Cafe-Filho, A.C. Management of wheat blast with synthetic fungicides, partial resistance and silicate and phosphite minerals. Phytoparasitica, Bet Dagan, v.42, n.5, p.609-617, 2014. Disponível em: $<$ http://link.springer.com/article/10.1007\%2Fs12600-014-0401-x. $>$ Acesso: 08out. 2015.

20. Reis, E.M.; Casa, R.T. Doenças dos cereais de inverno: diagnose, epidemiologia e controle. 2. ed. Lages, SC: Graphel, 2007. 176 p.

21. Rios, J.A.; Rios, V.S.; Paul, P.A.; Souza, M.A.; Neto, L.B.M.C.; Rodrigues, F.A. Effects of blast on components of wheat physiology and grain yield as influenced by fungicide treatment and host resistance. Plant Pathology, London, v. 66, n.6, p.877-889, 2017. Disponível em: <http://onlinelibrary. wiley.com/doi/10.1111/ppa.12634/pdf>. Acesso em: 08 jul. 2017

22. Rios, J.A.; Rios, V.S.; Paul, P.A.; Souza, M.A.; Araujo, L.; Rodrigues, F.A. Fungicide and cultivar effects on the development and temporal progress of wheat blast under field conditions. Crop Protection, Amsterdã, v. 89, n.1, p. 152-160, 2016. Disponívelem:<http://www.sciencedirect.com/science/ article/pii/S0261219416301776>Acesso em: 05 jan. 2017.

23. Rocha, J.R.A.S.C.; Pimentel, A.J.B.; Ribeiro, G.; Souza, M.A. de. Efficiency of fungicides in the control of wheat blast. Summa phytopathologica, Botucatu, v.40, n.4, p.347-352, 2014.

24. Silva, M.A.; Lima, G.S.A.; Assunção, I.P.; Barbosa, F.F.L.; Michereff, S. Progresso e arranjo espacial do mosaico dourado em fava. Revista Caatinga, Mossoró, v.28, n.1, p.19-27, 2015.

25. Silva, W.L; Cruz, M.F.A; Fortunato, A.A; Rodrigues, F.A. Histochemical aspects of wheat resistance to leaf blast mediated by silicon. Scientia Agrícola, Piracicaba, v.72, n.4, p.322-327, 2015.

26. Sousa, R.S.; Rodrigues, F.A.; Schurt, D.A.; Souza, N.F.A.; Cruz, M.F.A. Cytological aspects of the infection process of Pyricularia oryzae on leaves of wheat plants supplied with silicon. Tropical Plant Pathology, Brasília, DF, v.38, n.6, p.472-477, 2013.

27. Urashima, A.S.; Kato, H. Varietal resistance and chemical control of wheat blast fungus. Summa Phytopathologica, Jaguariúna, v.20, n.2, p.107112, 1994.

28. Urashima, A.S.; Lavorent, N.A.; Goulart, A.C.P; Mehta, Y.R. Resistence spectra of wheat cultivars and virulence diversity of Magnaporthe grisea isolates in Brazil. Fitopatologia Brasileira, Brasília, DF, v.29, n.5, p.511-518, 2004.

29. Urashima, A.S.; Grosso, C.R.F.; Stabili, A.; Freitas, E.G.; Silva, C.P.; Netto, D.C.S.; Franco, I.; MérolaBottan, J.H. Effect of M. grisea on seed germination, yield and quality of wheat. In: Wang, G.L.; Valent, B. eds. Advances in Genetics, Genomics and Control of Rice Blast Disease, Springer, Dordrecht, p. 267-277, 2009. Disponível em:<https://link.springer. com/chapter/10.1007/978-1-4020-9500-9 27>. Acesso em: 06 jul. 2015.

30. Zadoks, J.C.; Chang, T.T.; Konzac, C.F. A decimal code for the growth stages of cereals. Weed Research, Oxford, v.14, p.415-421, 1974. 